\title{
Towards an automated photogrammetry-based approach for monitoring and controlling construction site activities
}

\begin{abstract}
The construction industry has a poor productivity record, which was predominantly ascribed to inadequate monitoring of how a project is progressing at any given time. Most available approaches do not offer key stakeholders a shared understanding of project performance in real-time, which as a result fail to identify any project slippage on the original schedule. This paper reports on the development of a novel automatic system for monitoring, updating and controlling construction site activities in real-time. The proposed system seeks to harness advances in close-range photogrammetry to deliver an original approach that is capable of continuous monitoring of construction activities, with progress status determined, at any given time, throughout the construction lifecycle. The proposed approach has the potential to identify any deviation of as planned construction schedules, so prompt action can be taken because of an automatic notification system, which informs decision-makers via emails and SMS. This system was rigorously tested in a real-life case study of an in-progress construction site. The findings revealed that the proposed system achieved a significant high level of accuracy and automation, and was relatively cheap and easier to operate.
\end{abstract}

\section{Keywords: Photogrammetry, point cloud, BIM, construction site, monitoring, delays.}

\section{Introduction}

The majority of construction projects tend to suffer time and cost overruns. According to Companies' public annual reports (2013) 98\% of projects experience an average slippage of 20 months behind original schedule, and an average cost increase of $80 \%$ of original value. These overruns are associated with a failure to deal with loose control on projects, which are often brought about by poor project management strategies and old fashion technologies (Latham 1994; Kazaz et al. 2011). The conservative nature of the construction industry tends to cling to ineffective monitoring and controlling systems, which has severe consequences on the speed and robustness of decision-making (Langlois 1988; Jernigan 2008). The prevailing monitoring systems suffer from various inefficiencies that fail to detect potential delays. In addition, they do not have the ability to collect accurate data to reflect the correct as-built site progress status (Behnam et al. 2016).

Despite recent advances, the prevailing monitoring and management systems in the construction industry are still dominated by traditional approaches, including manual paperbased collection and recoding of on-site activities
(Kimoto et al. 2005; Yang et al. 2015). These approaches are often cumbersome, as site managers and inspectors manually collect and record progress of construction site activities, and then re-enter the collected records and interpret them at the site office (Kim et al. 2008). Moreover, this process is extremely slow as it takes approximately $20-30 \%$ of the feeders' daily efforts to update the construction activities (Golparvar-Fard et al. 2011; Solihin and Eastman 2015). This manualbased site monitoring and updating system has several limitations, such as missing, incomplete or incorrect information. Consequently, Project Managers (PM) commonly fail to obtain reliable progress details. This approach tends to lead to confusion, often leading PMs to misjudge the actual progress in their projects. Subsequently, unsound decisions are made, which has severe consequences on the effectiveness of the use of resources. For example, Kim et al. (2008) reported that, based on the manual progress monitoring system, a PM judged an activity to be only $30 \%$ finished, while in reality, it was $60 \%$ completed. In this case, the PM believed that the construction project was delayed, even though it was proceeding ahead of the planned schedule. Consequently, the PM deployed more resources than needed to that activity, which resulted in a waste of time and money. This demonstrates that current monitoring systems are often unreliable, time-consuming, 
costly, and prone to subjectivity and errors (Navon and Sacks 2007; Turkan et al. 2012).

To address construction projects' overrun, rigorous and reliable monitoring systems are needed to detect rapidly delays together with their root causes in order to alleviate them, once occurred (Mirahadi and Zayed 2016). Over the last few decades, a great deal of work sought to improve conventional monitoring, updating and controlling systems in the construction industry (Bosché 2012; Meža et al. 2014). In the same context, early efforts applied standalone technology to monitor and control construction site activities, but due to their inherent limitations, recent research sought to combine two or more technologies to improve the results of their monitoring systems.

\section{Current and emerging site monitoring and controlling systems}

Several attempts were made to resolve the salient challenges associated with the limitations of current monitoring and controlling systems. Consequently, a great deal of work has been devoted to develop monitoring technologies. Broadly speaking, these developments could be classified as standalone and integrated technologies.

\subsection{Standalone technology}

In this standalone proposed system only one technology is utilised (Navon 2000; Dick et al. 2004; Lukins and Trucco 2007; Bosché 2010). Earlier efforts by Navon (2000) focused on the development of a robotic system that could not only install tiles, but also monitor the site as it is equipped with cameras to measure the progress of the installed tiles. However, the robotic system required continuous human intervention for stabilisation and system calibration. Moreover, the robot was monochromatic, which in poor lighting conditions, especially indoors, affected adversely the accuracy of progress measurements. Therefore, the proposed robotic system lacked accuracy and reliability. It is worth mentioning that Navon (2000) himself considered the system incomplete and required further developments to minimise or eliminate human intervention.
A more developed automatic system proposed by Dick et al. (2004), produced an automatic framework acquisition for the 3D as-built model. The model was constructed from a small number of site photos by developing an algorithm that enabled recognition of the structural objects from the site photos. This framework succeeded to a limited extent to compare the as-built 3D model against the as-planned one. However, the results lacked the sufficient accuracy for site monitoring, as the optimum accuracy was $83 \%$ for vertical elements and $91 \%$ for horizontals.

A further advanced system proposed by Lukins and Trucco (2007) used Computer Vision (CV) to develop a classifier that can observe and detect changes (the progress status) during construction through a fixed camera. This was achieved by developing the prior building model and aligning it with the camera scenes to identify the progress. However, the system suffered from several limitations, including weather interference, occlusions, and daylight fluctuations. In addition, the whole system required continuous manual intervention. Consequently, the accuracy of the classifier is subject to the operator's accuracy and as a result makes the system prone to errors and time-consuming.

Software developers such as Autodesk tried to overcome the recognised limitations pertaining to conventional monitoring systems. Autodesk produced enabled a semi-automatic cloud based system to collect data from construction sites using Personal Digital Assistants (PDA) such as tablets or smartphones. Generally, PDAs overcame some of the recognised limitations, especially those related to the time required to collect data (Kim et al. 2008; Kimoto et al. 2005). However, the Autodesk's developed software/system still relied heavily on the inspectors to manually insert the construction site updates, which were not only subjective but also unreliable. Consequently, this method lacked the instant detection of delays, reliability and accuracy.

A more promising system proposed by Bosché (2010), sought to automate progress monitoring in construction sites by developing a point matching method using the Iterative Closest Point (ICP) recognition algorithm. This was achieved by using Laser Scanning (LS) technology to build a 3D point cloud model, which was then compared with the asplanned Building Information Model (BIM).

BIM involves the development and use of "a computer software model to simulate the 
construction and operation of a facility. The resulting model is a data-rich, object-oriented, intelligent and parametric digital representation of the facility, from which views and data are appropriated and analysed to generate information that can be used to make decisions and improve the process of delivering the facility" (Associated General Contractors of America AGC, 2005, p.3). Eastman et al. (2011) claimed that, using the BIM produced error-free design and boosted offsite prefabrication.

Bosché (2010) succeeded in achieving optimal registration and comparison between the project's Computer-Aided Design (CAD) model and the site LS model. The ICP approach was applied for comparison-based registration to estimate the differences between the two models. This system seemed promising but it relied heavily on manual interventions to perform synchronisation between the two models (i.e. as-built point cloud and asplanned models). Overall, this system is prone to human errors due to manual interventions, and is therefore time-consuming. Bosché (2010) himself considered the system as quasi automated. Above all, LS technology is not suitable for the majority of construction sites, due to its high costs, as well as the expertise needed for operation.

\subsection{Integrated technologies}

It is clear that standalone systems that use single technology are confronted with several limitations. Consequently, there has been a quest to integrate two or more technologies to combine their benefits, and to reduce the adverse effects of the standalone technology (El-Omari and Moselhi 2011; Ibrahim et al. 2009; Golparvar-Fard et al. 2009; Golparvar-Fard et al. 2011; Roh et al. 2011; Dimitrov and GolparvarFard 2014; Behnam et al. 2016).

The thrust of recent efforts shifted attention to mixed technologies to address the limitations of stand-alone technology. Accordingly, El-Omari and Moselhi (2011) proposed the integration between LS and photogrammetry to enhance the speed and accuracy of the acquired data from construction sites. The system succeeded in building a 3D asbuilt point cloud model with satisfactory accuracy by integrating LS and photogrammetry techniques, by synchronising the common points between the two point cloud models. Using the constructed 3D point cloud model, a comparison could be performed between the progress (as-built) model and the as- planned model. One of the main limitations of this system is the long time needed to perform a single scan. Indeed, to scan the entire built asset multiple moves are required from different positions, which make this system time-consuming and cumbersome. In addition, specialised technicians are often needed to perform the scans to collect accurate data. Above all, LS technique is still relatively expensive, which hampers its applicability for regular updates of construction site activities or to support timely and informed management decisions.

A similar system proposed by Ibrahim et al. (2009) relied on CV techniques to develop a progress monitoring system. This system analysed the geometric and material properties of the components in a BIM model and compared it with the corresponding elements from the collected site photos (the as-built). The comparison helped to identify the changes, reflected in the progress status of the construction site, which was then used to update the Work Breakdown Structure (WBS). Despite the promising results, the system suffered from several limitations, including the lack of sufficient details of the elements in the collected photos, which negatively affected the synchronisation with the BIM elements. Consequently, several elements could not be recognised, which had an impact on the accuracy and the reliability of the collected data.

Another integrated system developed by Golparvar-Fard et al. (2009) sought to automate monitoring the construction site activities by combining photogrammetry and time-lapse techniques to superimpose the as-built point cloud model constructed from the site photos over the asplanned 3D model, using the Augmented Reality (AR) technique. This system was able to depict the progress status in colour codes, where green meant as scheduled, dark green referred to ahead of schedule, and red depicted behind schedule. However, this monitoring system suffered from a mismatched level of details in the baseline schedule (as-planned), compared with the actual site. The proposed system could only recognise the completed activities with the same patterns and it overlooked the uncompleted/on-going activities. In addition, manual intervention was needed to filter and select the suitable photos, which made the process time-consuming, and highly dependent on the operator's experience and visual ability. Moreover, replicating the construction progress in a 
colour coding system was also deemed unsuitable for staff suffering from colour-blindness. A few years later, Golparvar-Fard et al. (2011) developed further the previous research for evaluating the status of the construction progress using the same approach of the colour-coding system. In this case, colour codes described the representation of time and cost values, as an analysis for Earned Value (EV). However, the later study did not address the other limitations of the Golparvar-Fard et al. (2009) proposal.

Another system, proposed by Roh et al. (2011), endeavoured to solve some of the weaknesses of previous systems by comparing the components of the as-built, abstracted from site photographs, against the as-planned BIM model. This was achieved by developing a classifier algorithm to synchronise the objects from BIM models with corresponding objects from captured photos. This system succeeded in providing the progress of construction elements in percentages. Due to significant manual intervention, critical data such as location and time was fed manually, which made it unreliable and inaccurate. In addition, training the classifier was an extremely time-consuming process, whereby the development of the algorithm required manual synchronisation of the construction elements, and thus the classifier accuracy heavily depended on human accuracy.

More recently, Dimitrov and Golparvar-Fard (2014) developed an algorithm that used a material texture recognition technique to automatically create 3D models by extracting the information from randomly collected site photos for construction elements (columns, walls, slabs, etc.). The automatically created 3D models were further used to depict the construction progress, after comparing them with the as-planned schedules. The proposed system was distinguished by its simplicity, but it was challenged for its inconsistent accuracy that affected its reliability. The system's accuracy varied from one material to another; hence the accuracy for the material recognition algorithm is $92.1 \%$ for casted concrete, and $92.3 \%$ for compacted soil. High accuracy was achieved in three elements only (formwork, grass and marble), but these elements are considered as minor elements in the construction industry (i.e. they usually have less impact on the critical path). In addition, the richness of the pre-prepared material recognition library heavily controlled the accuracy of the created $3 D$ model, thus some construction elements could not be recognised by the algorithm, leading to significant confusions in the constructed 3D model. The latest research by Behnam et al. (2016) proposed an automated system to generate and visualise the progress status of the repetitive construction activities for linear infrastructure projects. They integrated satellite remote sensing techniques and a Geographic Information System (GIS) web-based platform to build 3D models from satellite images and locations recognised by the GIS system. The accuracy of the created 3D model was improved by the collected site photos. Further comparison between the 3D created model and the 4D BIM model depicts the progress status in the form of visualised charts for easy understanding by PM and other stakeholders. The proposed automatic system succeeded, to a certain extent, to address some of the limitations of the previous automation attempts to monitor the progress of construction site activities. However, this system cannot be generalised to site activities, as it is limited to linear projects with repetitive activities, such as pipelines, road works, and railways. In addition, it did not provide sufficient information about start dates that needed to be inserted manually. Therefore, the system was deemed not fully automated as manual intervention is required to conjugate the geographical objects between the collected site photos and satellite images. Consequently, even for the longitudinal projects, the accuracy and the reliability of the progress update are still doubtful.

This review revealed that - to date - there is no approach/system that has successfully automated monitoring, analysing and controlling construction site activities to detect instantly any delays, once occurred. The aim of this study is to develop an original, close-range photogrammetry-based approach for monitoring and controlling construction site activities.

\section{Computer Vision based close-range photogrammetry}

CV based-metric cameras introduced significant technology that enabled the building of 3D models from 2D captured stills (Dong et al. 2016). In the last two decades, building accurate 3D models from 2D images is attracting a great deal of attention. The constructed 3D model is used in several fields such as monitoring construction activities (Kwak et al. 2013). 
Traditionally, Building 3D models from camera stills required network cameras to cover the target object(s) from different views (Detchev et al. 2014). The captured images used to construct 3D models that included enough details to measure the dimensions and the coordinates of the 2D images. Consequently, camera calibration process is the mainstay for correct data abstraction, as it enables the determination of the accurate relationship between each object in the photo and its physical representation (Zhang 2000; Dong et al. 2016; Percoco et al. 2017; Tan et al. 2017). Poor cameras' calibration may result in blemishes in images, such as distortions, which in turn can affect the reliability and accuracy of the abstracted information. Therefore, camera calibration tends to be the most crucial process for any computer vision application (Percoco et al. 2017; Fetić et al. 2012).

The purpose of this paper is to develop a novel automatic system that is able to promptly detect delays occurring in construction sites, and as a result send automatic notifications with progress updates to the concerned decision-makers, such as the PM and client.

\section{Methodology}

The proposed system is intended to be fully automated, which requires using a close-range photogrammetry technique to collect images from the construction site. These images are stored and fused together in one folder, based on the capturing date in a cloud server. The registered photos are exported to Agisoft PhotoScan pro software. This software has a built-in property to recognise and exclude any erratic photo(s). Subsequently, the selected photos will be used to construct a 3D point cloud model for a predetermined day/time figure $2 \mathrm{a}$ and $2 \mathrm{~b}$. The constructed 3D model encompasses the details to detect the discrepancies between the as-built and as-planned schedule, which be achieved by performing a comparison between the 3D point model and the BIM model. Subsequently, the system synchronised these discrepancies with the as-planned schedule to demonstrate the progress status. If the progress status complies with the as-planned program, notification is not required. However, if the system detects any deviation between the as-built (the progress status) and the as-planned, notification emails and SMS are sent to the concerned staff figure 1.

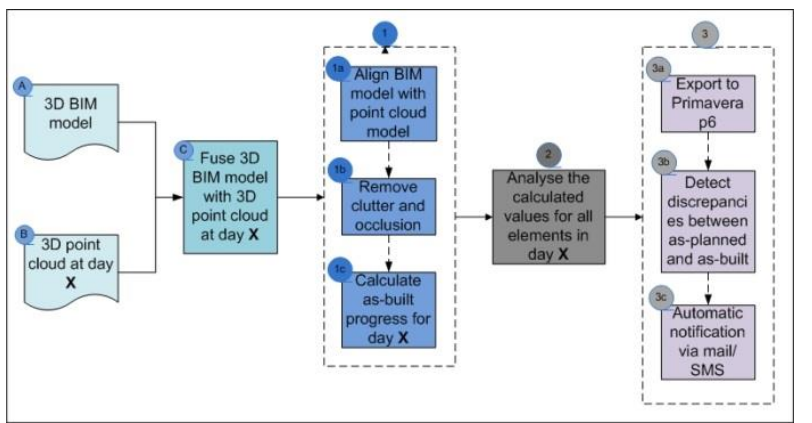

Fig. 1. Developed model to automate monitoring and controlling the construction site activities

To cover the details in the construction site activities, 12 cameras were installed in two different levels (i.e. 3 and $10 \mathrm{~m}$ height measured from the existing ground level). Cameras are installed on adjacent buildings and masts. However, the Wi-Fi internet router and Wi-Fi range extender are fixed on the adjacent buildings. Table 1 shows the specifications for the used cameras.

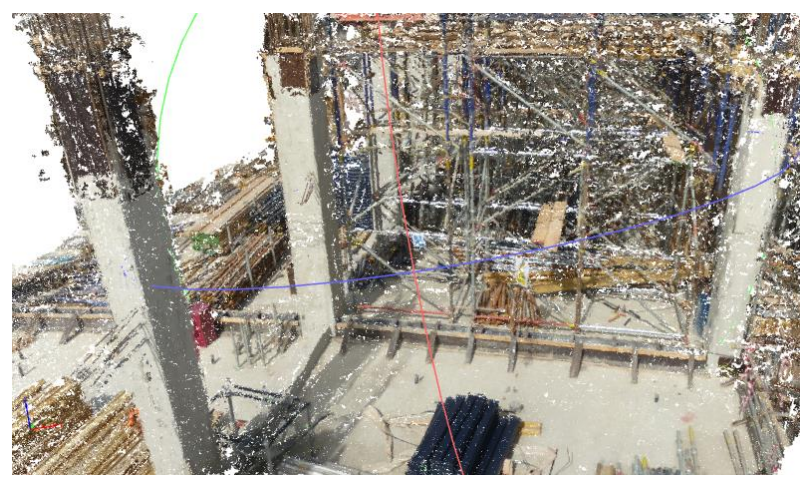

Fig. 2a. Point cloud built from captured photos for columns
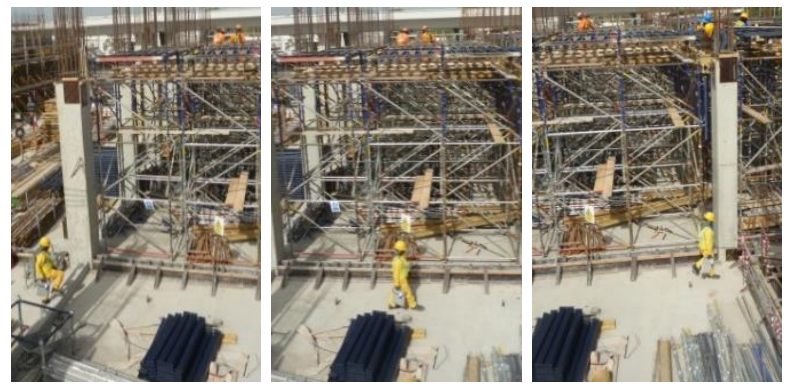

Fig.2b. Sample of captured site photos to construct point cloud model

Table 1

Camera specifications 


\begin{tabular}{|c|c|}
\hline Type & Specification \\
\hline Sensor & $\begin{array}{l}\text { 1" CMOS, Effective } \\
\text { pixels: } 20 \mathrm{M}\end{array}$ \\
\hline Lens & $\begin{array}{l}\text { FOV } 84^{\circ} 8.8 \mathrm{~mm} / 24 \mathrm{~mm} \\
\text { ( } 35 \mathrm{~mm} \text { format } \\
\text { equivalent) } \mathrm{f} / 2.8-\mathrm{f} / 11 \\
\text { auto focus at } 1 \mathrm{~m}-\infty\end{array}$ \\
\hline $\begin{array}{l}\text { Mechanical/electric } \\
\text { Shutter Speed }\end{array}$ & $8-1 / 2000 s ; 8-1 / 8000 s$ \\
\hline Shooting mode & $\begin{array}{l}\text { Auto+, Program } A E \text {, } \\
\text { Shutter priority } A E, \\
\text { Aperture priority } A E\end{array}$ \\
\hline $\begin{array}{l}\text { Wireless File } \\
\text { Transmitter }\end{array}$ & $\begin{array}{l}\text { Wireless File Transmitter } \\
\text { WFT-E7 }\end{array}$ \\
\hline Photo & $\begin{array}{l}\text { JPEG, DNG (RAW), } \\
\text { JPEG + DNG }\end{array}$ \\
\hline Battery life (CIPA) & 500 \\
\hline $\begin{array}{l}\text { Internet Wireless } \\
\text { Frequency Band }\end{array}$ & $2.4 \mathrm{GHz}$ \\
\hline $\begin{array}{l}\text { Min/Max Operating } \\
\text { Temperature }\end{array}$ & $-10^{\circ} \mathrm{C} / 50{ }^{\circ} \mathrm{C}$ \\
\hline $\begin{array}{l}\text { Remote Controller/ } \\
\text { Switch }\end{array}$ & $\begin{array}{l}\text { Remote control with N3 } \\
\text { type contact, Wireless } \\
\text { Controller LC-5, Remote } \\
\text { Controller RC-6 }\end{array}$ \\
\hline $\begin{array}{l}\text { Wi-Fi range } \\
\text { extender }\end{array}$ & $\begin{array}{l}\text { Wi-Fi range with speeds } \\
\text { up to } 2200 \mathrm{Mbps} \text { and } \\
\text { provides up to } 1000 \mathrm{M}^{2} \\
\text { Wi-Fi range }\end{array}$ \\
\hline
\end{tabular}

\subsection{Camera calibration}

Tan et al. (2017) concluded that there are two main techniques to calibrate cameras: (1) photogrammetric calibration, which requires prerequisite knowledge of the physical object such as its dimensions, coordinates and directions, in addition to the 2D information from the captured image. (2) Self-calibration does not require any prerequisite knowledge. Using the first approach gives more reliable and accurate data compared with the second one, because using advanced knowledge reduces the number of parameters (Tan et al. 2017). Therefore, this paper selected the photogrammetric calibration approach using the checkerboard $9 \times 6$ with a known pattern, size and structure.

To ensure cameras have the same geometrical representation of the captured scene, cameras were calibrated by shooting 32 images from different positions for the checkerboard figure 3. These photos were uploaded to MATLAB R2017b to determine the intrinsic (focal length, principal point, lens distortion coefficient) and extrinsic parameters of the cameras. In this process, once the 32 images were uploaded to MATLAB toolbox; the software recognised the corner points for the checkerboard figure 4. Subsequently, to determine the intrinsic and extrinsic parameters, MATLAB toolbox reprojected the corners to determine the re-projection errors figure 5a, (Fetić et al. 2012). During the calibration process, the following measures were considered; (1) optimum and clear view of the checkerboard, with minimum camera magnification to reduce blurred images (Percoco et al. 2017); (2) the Angle Of View (AOV) and Depth Of Field (DOF) were calculated to attain a minimum of $75 \%$ side overlap for consecutive shots; and (3) the control point pattern was well known and manufactured accurately. The calibration process was iterated a total of 6 times to acquire the best result by reducing the errors for the determined parameters figure $5 \mathrm{~b}$ and 5c. The following parameters were produced the best results to build the 3D model with accurate and reliable details: Focal length $45 \mathrm{~mm}$, Distance from object $27 \mathrm{~m}$, Angle for camera to capture photos $-45^{\circ}$ from the horizontal axes and Ground Sampling Distance (GSD) width is 0.1966 and height 0.1971 (cm/pixel).

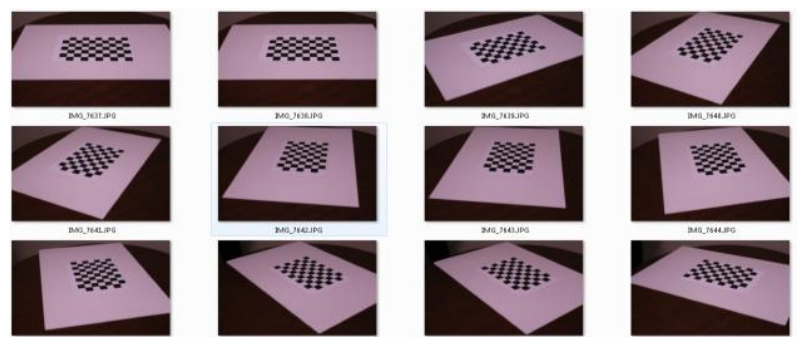

Fig. 3. Images captured for $9 \times 6$ planar checkerboard used for camera calibration 


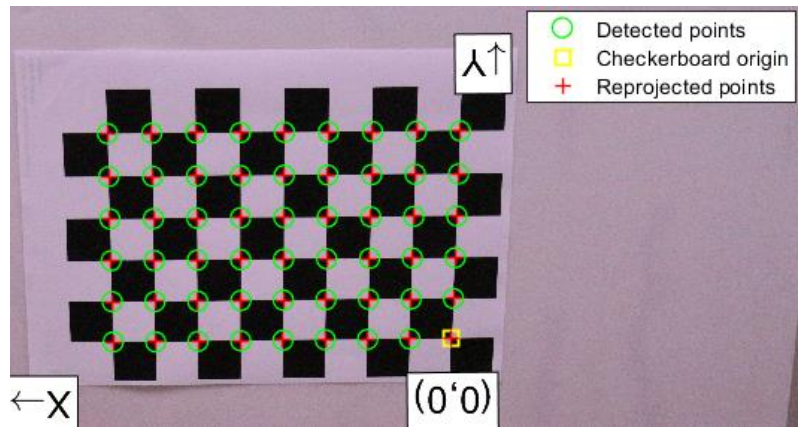

Fig. 4. The centre of the circle demonstrates images' detected corners, and the red cross intersection reprojected the corners using the calibrated camera parameters

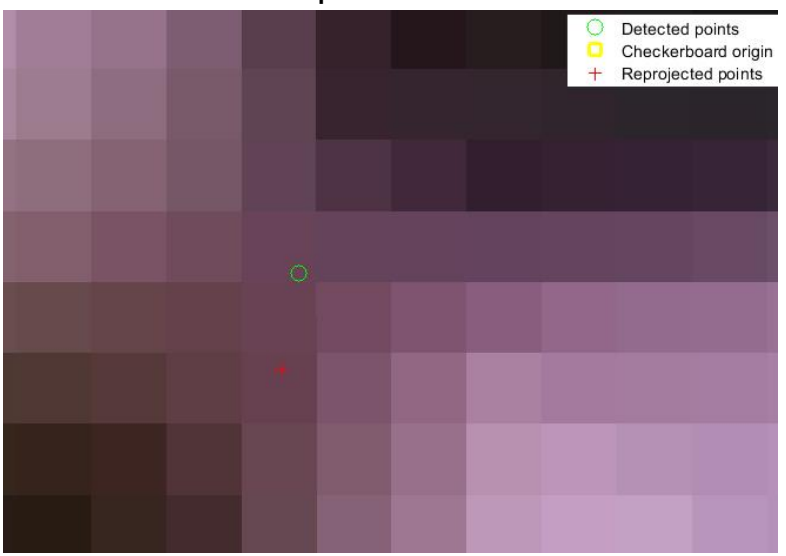

Fig. 5a. Reprojection error is the offset from the circle center to the cross intersection

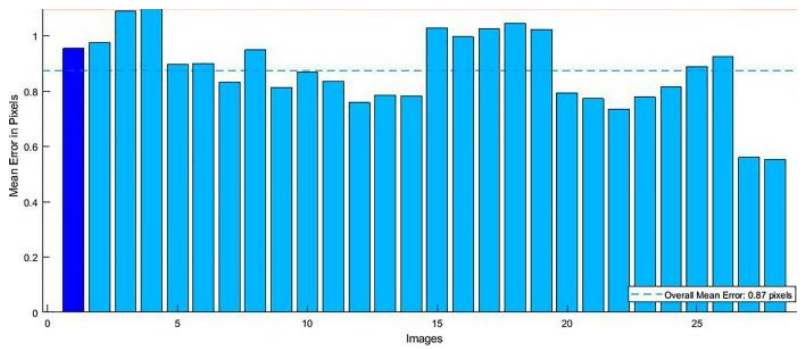

Fig. 5b: High reprojection mean error (0.87pixels)

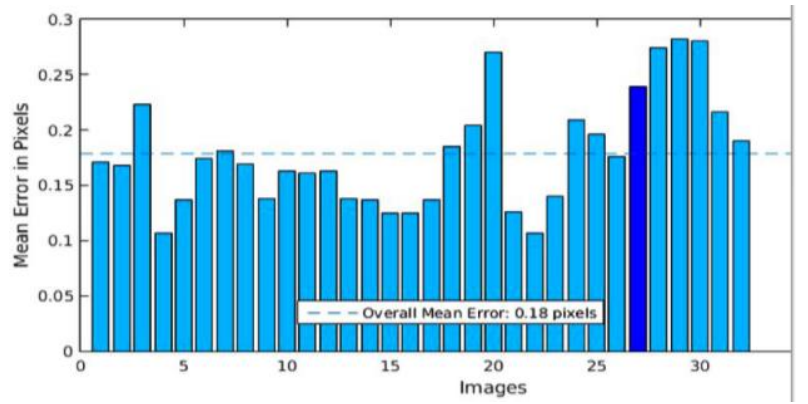

Fig.5c. Adjusted reprojection mean error $(0.18$ pixels)

\subsection{Case study}

To test the proposed system's validity and performance, it was rigorously tested in a real-life case study, to monitor and control the progress. Cameras were installed with precluded motion (i.e. cameras are static) to capture photos for the Reinforced Concrete (RC) columns. Only the RC columns superstructure was selected, mainly because these elements often lay on the critical path. Accordingly, any delay(s) for the superstructure were likely to affect the project adversely, thus leading to overruns.

Twelve cameras were installed and programmed to cover the tested elements and to capture a photo every 10 minutes for two hours at the end of each working day (i.e. starts at 5:00 PM till 7:00 PM). This is deemed as the best time to capture photos, because of lighting and glaring issues. In addition, it best captured progress as a cut-off date every day. Photos were taken with $75 \%$ side overlap for any two consecutive captured stills. Cameras were installed in a protected container following the Leung et al. (2008) container specification. The container reduced the impact from the local climate (i.e. temperature and humidity). In addition, it eliminated the negative effect on the captured images, mainly because of dirt accumulation on camera (Leung et al. 2008).

In order to automatically detect any discrepancies between as-planned and as-built progress status, four algorithms were developed.

\subsection{Algorithms to detect delays}

\subsubsection{Algorithm 1: registration of $3 D$ BIM model column surfaces}

The aim of this algorithm is to create internal and external boundaries/surface planes figure $6 \mathrm{a}$. And combine them into bounding boxes. The created bounding surfaces contain each column, so as to isolate the points relevant for each column to enable later processing. It uses the column surfaces modelled in the 3D BIM model in 6 directions (top, bottom, left, right, front and back), and then simply adds displacements of $5 \mathrm{~mm}$ to each in each direction (external and internal). The result is a pair of surfaces for each of the 6 sides, containing all relevant point cloud points. 


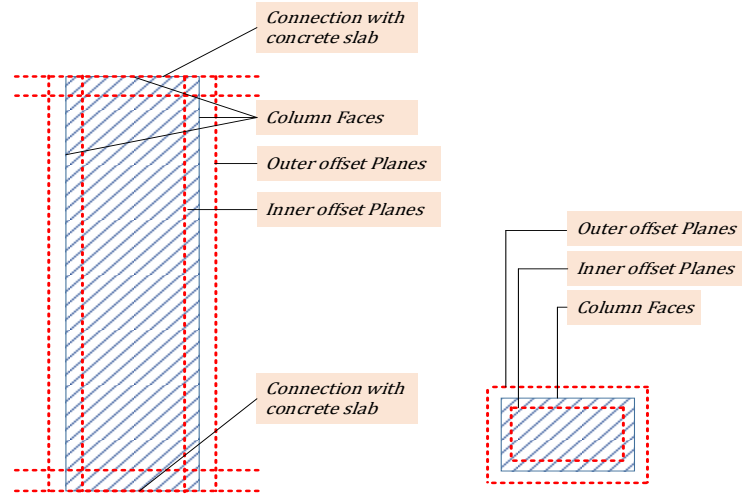

Fig.6a. creating virtual internal and external surface planes

\section{Algorithm 1:}

\section{Input}

- Column from 3D BIM model

- Column surfaces $(S 0, S 1, S 2 \ldots . . S 5)$

- Displacement offset distance Doffset

1. Recognise column original faces $(S 0, S 1, S 2 \ldots . . S 5)$

2. Add external planar surface surrounding the columns with offset $=$ Doff1

Doff $1=(\mathrm{Se} 0+5 \mathrm{~mm}, . . ., \mathrm{Se} 5+5 \mathrm{~mm})$

3. Identify intersections between planar surfaces (Se0, Se1,..., Se5)

4. Combine planar surfaces (Se0, Se1,..., Se5)

5. Add internal planar surface surrounding the columns with offset $D$ off 2

Doff2 $=($ SiO $+5 \mathrm{~mm}, \ldots$, Si5 $+5 \mathrm{~mm})$

6. Recognise intersections between planar surfaces (SiO, Si1,..., Si5)

7. Combine planar surfaces (Sio, Si1,.., Si5)

\section{Output}

- Column in 3D BIM model surrounded with additional external and internal surfaces

- External surfaces (Se0, Se1, Se2.... Se5) fig.6b

- Internal surfaces (SiO, Si1, Si2.... Si5) fig.6c

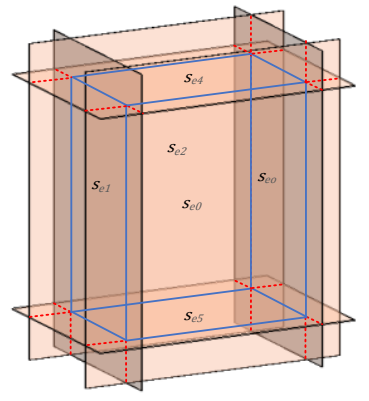

Fig.6b. Creating virtual external surface planers

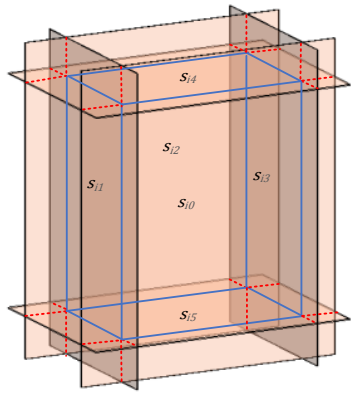

Fig.6c. Creating virtual internal surface planers

\subsubsection{Algorithm 2}

Aligning the 3D Point Cloud with the 3D Column from the BIM model

This algorithm seeks to align the two models i.e. the 3D point cloud model developed by Agisoft PhotoScan pro and 3D BIM model with external and internal surface planes from algorithm 1.

\section{Input}

- Internal offset planes (Sio, Si1, Si2,...., Si5)

- External offset planes (Se0,Se1, Se2,..., Se5)

1. Origin $A p j$ is the mean point in $3 \mathrm{D}$ $A p j=\frac{1}{n} \sum_{i}^{n}(X i, Y i, Z i)$

$n=$ number of points $\left(X i, Y_{i}, Z i\right)$ in point cloud shaping the column

2. Define column origin in BIM model $A m$

3. Translate the point cloud origin Apj to the column origin $\mathrm{Am}$

4. Compute transformation $(R, T)$

$R=3 D$ rotational angle

$T=3 D$ translation

5. Apply the transformation $(R, T)$ to the point cloud origin $A p j$ towards $A m$ in BIM model

6. Calculate error matrix

7. Iterate to minimise the error

8. Apply stopping criteria at error $\approx 0$

\section{Output}

- Aligned 3D point cloud to the 3D BIM model.

- Point Cloud Transformations $(R, T)$ are known.

\subsubsection{Algorithm 3: Determine column point cloud and remove occlusions}

This algorithm performed removal of clutters and occlusions to consider only the points which represent the as-built progress update for column(s). This algorithm filters the points in the point cloud by confining these points between the external and internal surface boundaries in algorithm 2.

\section{Algorithm 3}

Input

- External surfaces (Seo, Se1, Se2.... Se5)

- Internal surfaces (Sio, Si1, Siz.... Si5)

- Aligned point cloud $P c$ with 3D BIM model

1. $\left(P_{n}, P_{0}, P_{1}\right) \in P_{c}$ 
Pn Represents points outside the surface boundaries figure $6 \mathrm{~d}$

$P o$ Represents points located within the surface boundaries (between internal and external)

$P_{1}$ Represents points located within internal boundary only

2. For every point $p \in P_{c}$ :

If $p$ is between $S_{i 0}$ and $S_{e 0}$, or $S_{i 1}$ and $S_{e 1}$, or $\ldots$ $S_{e 0}$ and $S_{i 5}$

Register $p \in P_{0}$

3. For every point $p \in P_{c}$ :

If $p$ is between $S_{i 0}$ and $S_{i 5}$ (front and back internal surfaces)

Register $p \in P_{1}$

4. For every point $p \in P_{c}$ :

If $p \notin P_{1}$ and $p \notin P_{0}$

Register $p \in P_{n}$

5. Report column point cloud $P 0$, algorithm stops.

\section{Output}

- Confined point cloud Pobetween internal and external surface boundaries figure $6 \mathrm{~d}$.

- 3D point cloud with cleared from clutter and occlusion.

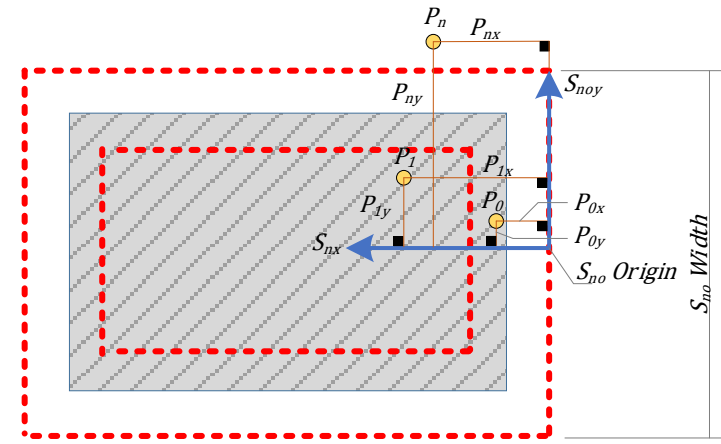

Fig.6d. Points are determined within the surface boundaries, according to algorithm 1

\subsubsection{Algorithm 4}

Calculation of the progreessive column volume The aim of this algorithm is to determine the asplanned concrete volume for each column, by measuring the true column heights $H$ (maximum heights) obtained from the point cloud in algorithm 3. To obtain the column's progressive volume arithmetic, multiplication $(H \times A)$ is applied where $A$ is the cross sectional area of the column. The algorithm starts by calculating the maximum height of the point cloud confined between the internal and external boundaries. Height calculation starts from the lower surface and progressively increases the height by a constant value of 10 millimeters. The existence and density of the points are then computed.

\section{Input}

- 3D BIM Column cross sectional area $A$

- Column with point cloud density $P O$

1. Algorithm starts by counting points of point cloud at

$\left\{h_{i}\right\}=\{0,10,20,30, \ldots . . m m\}$,

Where $h_{i+1}=h_{i}+10 \mathrm{~mm}$, representing height above a predefined coordinates of the column $(0,0,0)$.

2. Set $i=0$

3. Calculate point cloud density, $T_{i}$ in cross section at height $h_{i}$

4. Evaluate stopping criterion; $T_{i}<\varepsilon T_{i-1}$, where $\varepsilon=0.8$, indicating a sharp decrease in density due to $T_{i}$ being outside the body of the column.

5. If FALSE: set $i=i+1$; go to 3 . Else continue.

6. Set column height $H=h_{i-1}$

- The column height $H$ considered from the predefined coordinate $(0,0,0)$

7. Compute column volume $V j$

$V j=H \times A$

$A$ is the column's cross sectional area

$A=W \times L$

$W$ is the column width from BIM model

$L$ is the column length from BIM model

$\therefore V j=H \times W \times L$

\section{Output}

- Point cloud height represents the true progress of the concrete element.

- As-built progressive volume for any column.

\section{Results and discussion}

One of the most challenging issues encountered in the implementation of this system is related to occlusions. Occlusion is defined as any blockage of the camera vision by a physical object (Chi and Bisheng 2016). Occlusion can be classified into two main categories based on its source, static occlusion which is a result of a static object (such as scaffolding, steel rebar, timber, etc.), and dynamic occlusion which is a result of movable objects (such 
as labourers, machines, etc.). Indeed, it is extremely difficult to obtain a clear image without occlusions figure 7. On a construction site, occlusions are often inevitable (Dimitrove and Gloparvar-Fard 2014; Ibrahim et al. 2009).

To reduce dynamic occlusion it was decided to capture site photos after the duty time (i.e. after 5:00 $\mathrm{pm})$. The selected time significantly reduced the dynamic occlusions for captured photos because the site is shut-down; accordingly there are no active labourers or machines. Although $60 \%$ of the side overlap is Agisoft PhotoScan Pro recommendation, it was decided to consider $75 \%$ for side overlap for captured photos to ensure the same object has at least 3 photos from different viewing positions. This process enabled the software to remove automatically any dynamic occlusion by applying simple background-foreground subtraction from the 2D images (Nguyen and Smeulders 2006; Chi and Bisheng 2016; Sengar and Mukhopadhyay 2017; Agisoft PhotoScan User Manual 2017).

However, static objects usually occlude camera vision in two forms either partially or fully. Full occlusion is defined as any action that precludes camera to capture photo(s) for the target object (Chi and Bisheng 2016). Full occlusion was deemed beyond the scope of this research, as monitoring construction sites is largely concerned with partial occlusions. The proposed system put forward a technique to reduce the impact of the partial static occlusion that usually occurs in construction sites, especially for vertical elements. Whereas - by default - the progress for concrete elements, such as columns, is vertical, and the cross-sectional area is known from the BIM model. As a result, the most important part for the camera to capture is the casted height; which is obtained by applying algorithm 3 and 4 . The outputs from algorithm 4 are effective even with partial occlusion, provided that the last part of the element (i.e. the column tip) is free from occlusions.

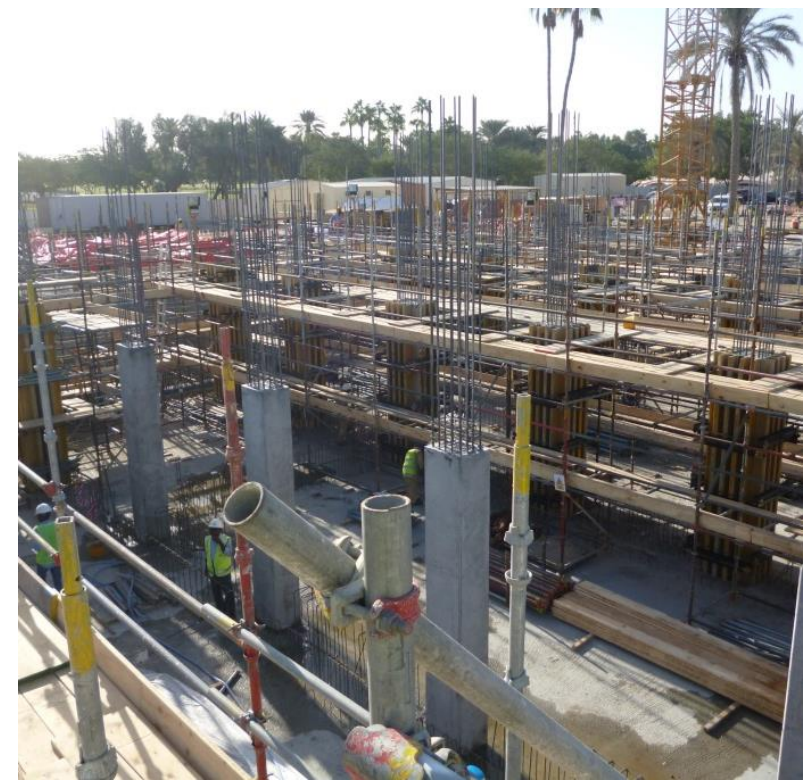

Fig.7. Construction site image shows static occlusions (i.e. scaffolding) due to daily construction activities

As a result, each element/column is given a unique code in accordance with the baseline program coding system, as shown in figure 8 . The differences between the 3D point cloud and BIM models (the outputs) are the as-built progress status, in terms of units $\left(\mathrm{m}^{3}, \mathrm{~m}^{2}, \mathrm{~m}\right.$ or number). These outputs are sent automatically to the asplanned program to determine the progress update.

If as-built and as-planned are similar, it means that there is no deviation. However, if any deviation is detected due to mismatches between the asplanned and the as-built, automatic notifications are sent to appropriate decision-makers. Mismatches refer to any differences detected in the structure's dimensions, while comparing the point cloud model with the BIM model. The system considers "deviation" as any activity ahead or delayed when compared with the as-planned schedule. Once the progress status is updated, the notification automatically alerts the concerned parties via SMS figure $9 \mathrm{a}$ and/or emails figure $8 \mathrm{~b}$. The proposed system supports decision-makers to obtain timely and accurate information, which assist them to make the right decisions at the right time to address any potential project overruns. 


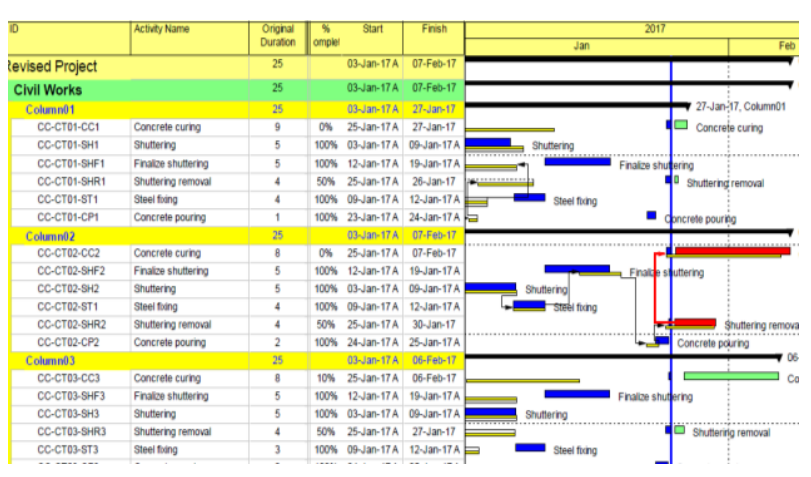

Fig.8. Construction program for the project (the as-planned program)

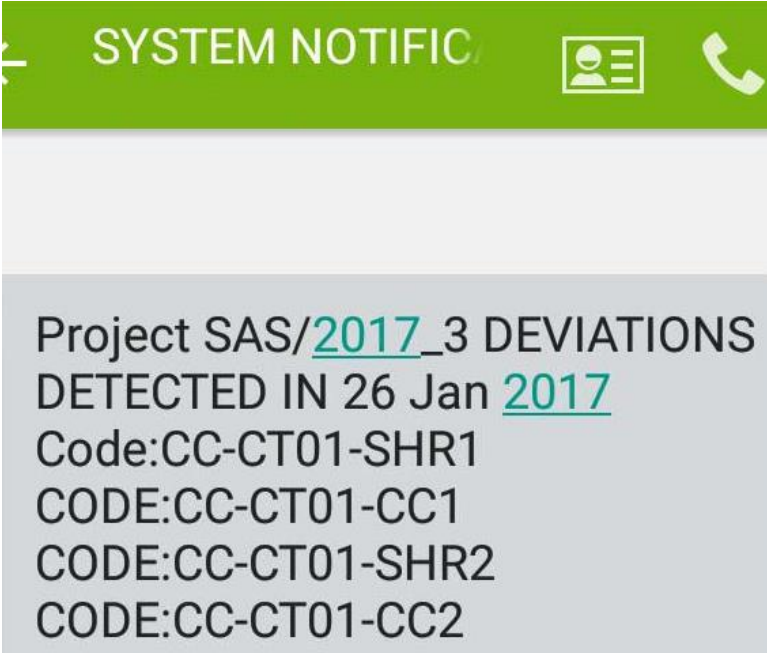

ETI...LAT $8: 46 \mathrm{pm}$

Fig.9a. SMS sent automatically to notify the project manager for the deviated activities detected by the system

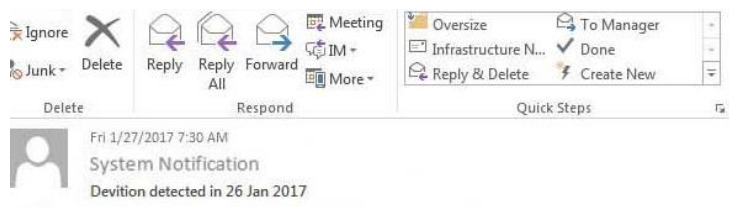

Project SAS/2017_3

Devition detected in 26 Jan 2017

Code: CC-CT01-SHR1

Code:CC-СT01-CC1

Code:CC-CT01-SHR2

Code: CC-CT01-CC2

Please note this is an auto generated e-mail. Please do NOT reply to this email

Fig.9b. Email sent automatically to notify the project manager for the deviated activities detected by the system

\subsection{System's accuracy}

The case study revealed that the whole process starting from the initial storing of the photos until the sending of notification emails and SMS takes less than 60 minutes. Details of the accuracy obtained from the case study are shown in table 2. The recorded error for the horizontal dimensions is 0 $\mathrm{mm}$, which means that a horizontal accuracy of $100 \%$ was achieved, because data was abstracted from the BIM model. Similarly, the accuracy for the vertical dimensions is $6 \mathrm{~mm}$ for every 1 linear meter, resulting in a vertical accuracy of $99.4 \%$. However, the accuracy for the areas and volumes is $100 \%$ and $99.99 \%$ respectively, and $100 \%$ accuracy was achieved for quantified numbers.

Table 2

System's accuracy

\begin{tabular}{llc}
\hline$\%$ & Error $(\mathrm{mm})$ & Accuracy \\
\hline Horizontal & $0 \mathrm{~mm} / 1 \mathrm{~m}$ & $100 \%$ \\
\hline Vertical & $6 \mathrm{~mm} / 1 \mathrm{~m}$ & $99.4 \%$ \\
\hline Area & $0 \mathrm{~mm}^{2} / 1 \mathrm{~m}^{2}$ & $100 \%$ \\
\hline Volume & $6 \mathrm{~mm}^{3} / 1 \mathrm{~m}^{3}$ & $99.99 \%$ \\
\hline Quantified number & zero & $100 \%$ \\
\hline
\end{tabular}

These results are significant and suggest that the proposed system has delivered a step change in improving the monitoring, analysing, updating, and controlling construction site activities.

The proposed approach deemed rigorous as the achieved accuracy, reported in table 2, is better than any other proposed system to date, wherein only Dimitrov and Golparvar-Fard (2014) achieved 100\% accuracy for three suboptimal construction elements (i.e. formwork, grass and marble). Table 3 demonstrates the high level of accuracy achieved by this proposed system in comparison to previous/available systems.

Table 3

Accuracy of proposed system in comparison to other systems

\begin{tabular}{cccc}
$\frac{5}{0}$ & $\begin{array}{c}\text { Used } \\
\frac{\text { Technology }}{\sigma}\end{array}$ & $\begin{array}{c}\text { Accuracy for } \\
\text { construction } \\
\text { elements }\end{array}$ & Authors \\
$\frac{\varepsilon}{0}$ & & \\
$\frac{0}{3}$ & & \\
\hline
\end{tabular}




\begin{tabular}{|c|c|c|c|c|}
\hline 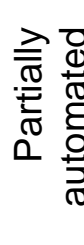 & $\begin{array}{l}\text { framework } \\
\text { acquisition } \\
\text { and } \\
\text { photogramm } \\
\text { etry }\end{array}$ & Horizontal & $\begin{array}{l}\stackrel{\circ}{\circ} \\
\infty \\
\circ \\
\frac{\circ}{\sigma}\end{array}$ & $\begin{array}{c}\text { Dick et al. } \\
\quad(2004)\end{array}$ \\
\hline 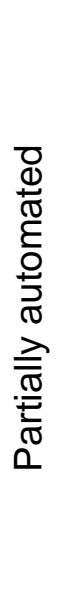 & $\begin{array}{l}\text { Material } \\
\text { texture } \\
\text { recognition } \\
\text { and } \\
\text { photogramm } \\
\text { etry }\end{array}$ & $\begin{array}{l}\text { Compacted } \\
\text { soil } \\
\text { Formwork, } \\
\text { grass, } \\
\text { marble }\end{array}$ & 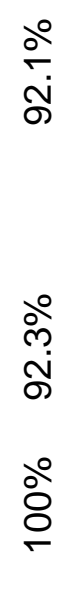 & $\begin{array}{l}\text { Dimitrov } \\
\text { and } \\
\text { Golparvar- } \\
\text { Fard } \\
(2014)\end{array}$ \\
\hline $\begin{array}{l}\overline{0} \\
\frac{d}{\pi} \\
\stackrel{0}{E} \\
0 \\
\frac{0}{3} \\
\frac{0}{3} \\
\overline{1}\end{array}$ & $\begin{array}{l}\text { Photogramm } \\
\text { etry, BIM and } \\
\text { point cloud }\end{array}$ & $\begin{array}{c}\text { Numbers } \\
\text { Areas }\end{array}$ & 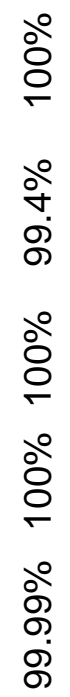 & $\begin{array}{l}\text { Proposed } \\
\text { system }\end{array}$ \\
\hline
\end{tabular}

The proposed approach is fully automated and does not require any expertise to operate the system. The findings revealed that the proposed system is original and could significantly improve construction site monitoring and controlling.

\section{Limitations of the system and further study}

The only human intervention required is to install and program the cameras, but future developments in photogrammetry could address this issue. In addition, the batteries of the cameras needed to be replaced every 37 days.
Although the system was tested for one construction element only i.e. the RC columns, the accuracy and performance of the system for multiple construction elements warrant further investigation. This paper assumed all construction elements implemented according to the BIM model only. Finally, the system has been tested for outdoor construction activities only. Future research will test the system for other construction elements and under different conditions (i.e. indoor environment).

\section{Conclusion}

This paper reported the development of state of the art automated close range photogrammetrybased approach for monitoring and controlling construction site activities. The case study demonstrated that the proposed system was novel and highly rigorous in automatically monitoring, analysing, updating and notifying the progress status to decision-makers. In particular, the system succeeded to instantly detect any discrepancies from the as-planned schedule to provide various stakeholders with accurate and timely feedback. This is likely to support decision-makers to take the appropriate decisions based on authoritative data with a significantly high level of accuracy. Thanks to this system, a horizontal accuracy of $100 \%$ was achieved, while the vertical accuracy exceeded $99.4 \%$.

Once cameras are installed and programmed, the system does not require any expertise or manual intervention. Above all, the system is easy to use and much cheaper to operate. In addition, it can be used for any outdoor construction project. The progress status can be obtained anytime and anywhere.

\section{References}

Agisoft LLC (2017). Agisoft PhotoScan user manual: Professional Edition, version 1.3 [Accessed in 2 January 2018] Available at: http://www.agisoft.com/pdf/photoscanpro 13 en.pdf

American Institute of Architects (2002), the architect's handbook of professional practice, (New York, John Wiley and Sons, Inc. 
work package components in construction projects using computer vision, Journal of advanced engineering informatics, vol. 23, pp. 93-103. https://doi-

org.ezproxy.uwe.ac.uk/10.1016/i.aei.2008.07.002

Jernigan, F (2008). Big BIM, little bim: the practical approach to building information modeling: integrated practice done the right way! 2nd edn, 4Site Press, Salisbury, MD.

Kazaz, A., Ulubeyli, S. and Tuncbilekli, N (2011). Causes of delays in construction projects in turkey, Journal of civil engineering and management, vol.18 (3), $\quad$ pp. 426-435. http://dx.doi.org.ezproxy.uwe.ac.uk/10.3846/13923 $\underline{730.2012 .698913}$

Kim, Y., Oh, W., Cho, K. and Seo, W (2008). A PDA and wireless web-integrated system for quality inspection and defect management of apartment housing projects, Automation in construction, vol. 17(2), pp.

163-179.

https://doi.org/10.1016/i.autcon.2007.03.006

Kimoto, K., Endo, K., Iwashita, S. and Fujiwara, M (2005). The application of PDA as a mobile computing system on construction management, Automation in construction, vol.14, pp. 500-511. https://doi.org/10.1016/j.autcon.2004.09.003

Kwak,E., Detchev,I., Habib, A., El-Badry,M and Hughes, C (2013). Precise photogrammetric reconstruction using model-based image fitting for 3D beam deformation monitoring. Journal of surveying engineering, vol. 139 , pp. 14

-155. $\quad$ https://doi.org/10.1061(ASCE)SU.1943$\underline{5428.00001 \overline{05}}$

Langlois, $R$ (1988). NATIONAL RESEARCH COUNCIL. Toward a new era in U.S. manufacturing: The need for a national vision (Book Review), American Economic Association, Nashville, Tenn, vol 26(1), p.137

Latham, M (1994). Constructing the team: joint review of procurement and contractual arrangements in the United Kingdom construction industry: final report. London. Department of the Environment [online]. London: [Accessed 1 August 2017]. Available
content/uploads/Constructing-the-team-TheLatham-Report.pdf

Leung, S., Mak,S. and Lee,B (2008). Using a realtime integrated communication system to monitor the progress and quality of construction works, Automation in construction, vol.17,pp.749-757. https://doi.org/10.1016/j.autcon.2008.02.003

Lukins, T. and Trucco, E (2007). Towards Automated Visual Assessment of Progress in Construction Projects, British Machine Vision Conference, pp. 1-10. [Accessed in 4 August 2017] http://ai2-s2-

pdfs.s3.amazonaws.com/128b/8e3d93daf8ecbc5ca c196939dde7cf6d9822.pdf

Meža, S., Turk, Z. and Dolnec, M (2014). Component based engineering of a mobile BIMbased augmented reality system, Automation in construction,vol. 42(1), pp. 1-12. https://doi.org/10.1016/j.autcon.2014.02.011

Mirahadi, F. and Zayed, T (2016). Simulation-based construction productivity forecast using NeuralNetwork-Driven Fuzzy Reasoning, Automation in Construction, vol. 65, pp.102-115. https://dx.doi.org/10.1016/j.autcon.2015.12.021

Navon, R (2000). Process and quality control with a video camera, for a floor-tiling robot, Automation in Construction, vol.10 (1), pp. 113-125.

Navon, R. and Sacks ,R (2007). Assessing research issues in Automated Project Performance Control (APPC), Automation in construction, vol.16(4), pp. 474-484. https://doi.org/10.1016/S0926$\underline{5805(99) 00044-8}$

Nguyen, Hieu and Smeulders, A (2006). Robust tracking using foreground-background texture discrimination, international journal of computer vision, vol. $69 \quad$ (3), pp. 277-293 https://dx.doi.org/10.1007/s11263-006-7067-x

Omar, H. and Dulaimi, M (2015). Using BIM to automate construction site activities, Proceeding of conference Building Information Modeling (BIM) in design, construction and operations, Bristol, UK. vol.149, pp. 45-58.

https://doi.org/10.2495/BIM150051 at:http://www.cewales.org.uk/cew/wp- 
Percoco, G., Guerra, M., Salmeron,A and Galantucci, L (2017). Experimental investigation on camera calibration for 3D photogrammetric scanning of micro-features for micrometric resolution, International journal of advanced manufacturing technology, vol. 91(9), pp. 29352947. https://doi.org/10.1007/s00170-016-9949-6

Roh, S., Aziz, Z. and Pena-Mora, F (2011). An object-based 3D walk-through mode for interior construction progress monitoring, Automation in construction, vol.20, pp. 66-75. https://doi.org/10.1016/j.autcon.2010.07.003

Sengar, S and Mukhopadhyay, S (2017). Foreground detection via background subtraction and improved three-frame differencing, Arabian Journal for Science and Engineering, vol. 42(8) pp. 3621-3633. https://doi.org/10.1007/s13369-0172672-2

Solihin, W. and Eastman, C (2015). Classification of rules for automated BIM rule checking development, Automation in construction, vol.

Tan, L., Wang, Y., Yu, H and Zhu, J (2017). Automatic camera calibration using active displays of a virtual pattern, IEEE, pp. 1416-1428

\section{https://doi.org/10.1109/ICIEA.2016.7603807}

Turkan, Y., Bosche, F., Haas, C. and Haas, R (2012). Automated progress tracking using 4D schedule and 3D sensing technologies, Automation in construction, vol.22, pp. 414-421. https://doi.org/10.1016/i.autcon.2011.10.003

Yang, J., Park,W., Vela, P. and Golparvar-Fard, M (2015). Construction performance monitoring via still images, time-lapse photos, and video streams: Now, tomorrow, and the future, Advanced engineering informatics, vol. 29(2), pp.211-224. https://doi.org/10.1016/.aei.2015.01.011

Zhang, Z (2000). A flexible new technique for camera calibration, IEEE transaction on pattern analysis and machine intelligence, vol. 22(11), pp. 1330-1334. https://doi.org/10.1109/34.888718 